\title{
Sensitivity and Specificity of the Fever Syndromes in BioSense and ESSENCE
}

\author{
Caleb Wiedeman* \\ Tennessee Department of Health, Nashville, TN, USA
}

\section{Objective}

To objectively compare the BioSense and ESSENCE fever syndromes using recorded temperature as a gold standard.

\section{Introduction}

Syndromic surveillance refers to the monitoring of disease related events, sets of clinical features (i.e. syndromes), or other indicators in a population. Tennessee obtains emergency department data for syndromic surveillance in standardized HL7 format following the field and value set standards published by the Public Health Information Network. Messages contain information previously unavailable to syndromic surveillance systems, including quantitative values such as recorded temperature. Data are received daily and processed by a Tennessee ESSENCE application and the national BioSense platform.

These systems use chief complaint keywords, ICD9 codes, and other algorithms to assign syndromes for each record. The differences between the BioSense and ESSENCE syndrome assignments have not been well defined. Detailed comparisons of syndrome assignment across tools are difficult to perform due to the intensity of the manual review required. However, definitions of fever can be easily confirmed in HL7 messages when the recorded temperature is provided. Currently, both the BioSense and ESSENCE syndrome definitions exclude recorded temperature from consideration when assigning syndromes.

To compare the performance of the fever syndromes used by BioSense and ESSENCE, recorded temperature data was used as the gold standard.

\section{Methods}

Emergency department data from five Memphis area hospitals during 7/1/14 - 6/30/15 were used for this analysis. BioSense data were queried and extracted using the system supplied version of RStudio and analyzed using SAS 9.4. A "visit" was defined a record with a unique combination of facility and the BioSense created variable "analysis visit id". Data lacking a syndrome assignment were excluded from the analysis. Temperature was determined by taking the maximum recorded temperature (Tmax) across all updates for each record.

ESSENCE overwrites records with each update, so recorded temperature at the last update was used as Tmax for this evaluation. A "true" fever was defined as any record with a Tmax of greater than 100 degrees Fahrenheit. Sensitivity, specificity, positive predictive value, and negative predictive value were calculated for the fever syndrome in BioSense and ESSENCE.

\section{Results}

There were 326,966 records assigned to a syndrome with recorded temperature identified in the BioSense data $(18,744$ observations were missing temperature) and 319,246 records identified in the ESSENCE data (27,237 missing temperature). Sensitivity and specificity are plotted month by month in Figure 1. Positive predictive value and negative predictive value were not substantially different.

\section{Conclusions}

The BioSense fever syndrome and the ESSENCE fever syndrome performed similarly. ESSENCE had higher sensitivity compared with the BioSense fever syndrome throughout the study period.

There were some discrepancies in both data sets. The small difference in total visit numbers across both systems is likely due to additional processing rules in the BioSense platform. Also, ESSENCE had a larger number of observations missing temperature, likely due to record updates overwriting existing temperature data with missing data. Theoretically, update messages sent via HL7 should contain all information sent previously, but this may not be true for recorded temperature data. Additional investigation is needed to see if the overwrites are true corrections by the submitting facility or attributable to another source.

Recorded temperature adds value to syndromic surveillance practice when sent consistently. Including recorded temperature data as a queryable variable would increase the sensitivity of the fever syndromes in both ESSENCE and BioSense.

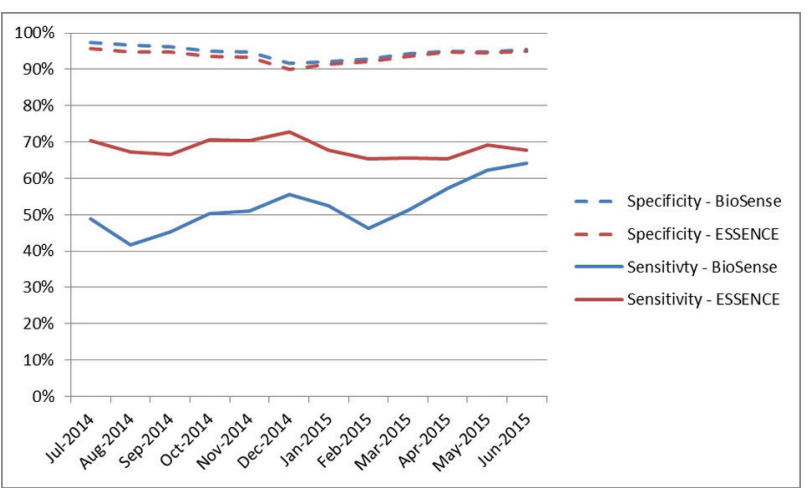

Figure 1: Sensitivity and Specificity of the BioSense and ESSENCE Fever Syndromes from July 2014 through June 2015

\section{Keywords}

BioSense; ESSENCE; fever; syndrome

\section{${ }^{*}$ Caleb Wiedeman}

E-mail: caleb.wiedeman@tn.gov 